\title{
Erratum: Light quark mediated Higgs boson threshold production in the next-to-leading logarithmic approximation
}

\author{
Charalampos Anastasiou ${ }^{a}$ and Alexander Penin ${ }^{b}$ \\ ${ }^{a}$ Department of Physics, University of Alberta, \\ Edmonton AB T6G 2J1, Canada \\ ${ }^{b}$ Institute for Theoretical Physics, ETH Zürich, \\ 8093 Zürich, Switzerland \\ E-mail: babis@phys.ethz.ch, penin@ualberta.ca
}

ERRATUM TO: JHEP07(2020)195

ArXiv EPrint: 2004.03602

In the paper a NLL contribution of the gluon vertex correction figure 4(c) has been omitted. Including this contribution results in the following corrections. In the integrand of eq. (3.5) $\eta+\xi$ should be replaced with $4 \eta+4 \xi-2$. In eqs. (3.5), (3.6) $g_{\gamma}(x)$ should be replaced with $4 g_{\gamma}(x)-g(x)$. In eq. (3.7) the coefficients of $C_{F} x$ and $C_{F} x^{2}$ terms should be corrected from $1 / 5$ and $1 / 35$ to $3 / 10$ and $1 / 21$, respectively. In the second and third lines of eq. (4.2) the coefficients of $C_{F}$ terms should be corrected from 1 and $1 / 7$ to $3 / 2$ and $5 / 21$, respectively. In the text below eq. (4.2) the coefficient $\left(C_{A}-C_{F}\right)\left(11 C_{A} / 9-3 C_{F} / 2-2 T_{F}\right) / 640$ should be corrected to $\left(C_{A}-C_{F}\right)\left(11 C_{A} / 9-C_{F}-10 T_{F} / 3\right) / 640$. The numerical values of the NNLO and $\mathrm{N}^{3} \mathrm{LO}$ NLL corrections in table 1 should be changed from -2.170 and -2.189 to -2.183 and -2.204 , respectively. After the correction the abelian part of our result agrees with the results [1-3] for the Higgs boson two-photon decay amplitude. The numerical impact of the correction on the threshold cross section in NNLO and $\mathrm{N}^{3} \mathrm{LO}$ is very small.

Open Access. This article is distributed under the terms of the Creative Commons Attribution License (CC-BY 4.0), which permits any use, distribution and reproduction in any medium, provided the original author(s) and source are credited. 


\section{References}

[1] Z.L. Liu, B. Mecaj, M. Neubert and X. Wang, Factorization at Subleading Power and Endpoint Divergences in Soft-Collinear Effective Theory, arXiv:2009.04456 [INSPIRE]

[2] Z.L. Liu, B. Mecaj, M. Neubert and X. Wang, Factorization at Subleading Power and Endpoint Divergences in $h \rightarrow \gamma \gamma$ Decay: II. Renormalization and Scale Evolution, JHEP 01 (2021) 077 [arXiv: 2009.06779] [INSPIRE].

[3] M. Niggetiedt, Exact quark-mass dependence of the Higgs-photon form factor at three loops in $Q C D$, arXiv:2009.10556 [INSPIRE]. 